\title{
Análisis de la disposición técnica de los vehículos automotores como vía de valoración del fin de vida útil
}

\author{
Laksmi Penabad Sanz ${ }^{1}$, Pedro Antonio Rodríguez Ramos ${ }^{2}$
}

\begin{abstract}
${ }^{1,2}$ Centro de Estudios de Ingeniería en Mantenimiento, Facultad de Ingeniería Mecánica, Universidad Tecnológica de La Habana José Antonio Echeverría. Calle 114 \#11901, entre 119 y 127, Cujae, Marianao, La Habana, Cuba.

Email: laksmi@mecanica.cujae.edu.cu,parr@ceim.cujae.edu.cu

Received: January $23^{\text {th }}, 2018$.

Accepted: February $25^{\text {th }}, 2018$.

Published: March $30^{\text {th }}, 2018$.

Copyright (C2016 by authors and Institute of Technology Galileo of Amazon (ITEGAM).

This work is licensed under the Creative Commons Attribution International

License (CC BY 4.0).

http://creativecommons.org/licenses/by/4.0/ (c) (1) (3) (2) Open Actes:

ABSTRACT

The production chain for the treatment of vehicles at the end of life is very important to any country, both economically and environmentally. The decision to end the life of vehicles must be taken at the right time in order to get the smallest economic and environmental negative effects to the vehicles operation. This paper proposes that this assessment, in the case of transport fleets, starts from the identification of vehicles which operation is irrational. This identification can be obtained comparing the vehicles readiness with the minimum permissible readiness. For this, the boundary condition of fleet rational use is defined and integrated with the equation for calculating the indicator Readiness. This allows obtaining a function whose parameters depend on the specific technical and economic characteristics where transportation activity occurs so the reference value for the comparison is determined. It also shows the application to a real case that allowed to identify vehicles whose operation is irrational and then to evaluate whether to pass to the supply chain for end of life vehicles.
\end{abstract}

Keywords: Readiness, End of vehicles' life, rational use, motor vehicles.

\section{Analysis of the motor vehicles readiness as a means of end of life assessment}

\begin{abstract}
RESUMEN
La cadena productiva para el tratamiento de los vehículos al final de su vida útil es de gran importancia para cualquier país, tanto desde el punto de vista económico como medioambiental. La decisión de dar por terminada la vida útil de vehículos debe tomarse oportunamente, de manera que las afectaciones económicas y medioambientales de la explotación de los vehículos sean menores. El presente trabajo propone que esta valoración, en el caso de las flotas de transporte, parta de la identificación de los vehículos cuya explotación es irracional. Esta identificación se puede obtener como resultado de la comparación de la disposición técnica de los vehículos con la disposición técnica mínima permisible. Para ello se define la condición límite de uso racional de las flotas y se integra con la ecuación de cálculo del Coeficiente de Disposición Técnica. Esto permite obtener una función cuyos parámetros dependen de las características técnico-económicas concretas donde se desarrolla la actividad de transportación con lo que se determina el valor de referencia para la comparación. Asimismo, se muestra la aplicación a un caso de estudio que permitió identificar los vehículos cuya explotación es irracional y de esta manera decidir, luego del análisis correspondiente, la conveniencia de darles baja y transitar a la cadena productiva para el tratamiento de fin de vida.
\end{abstract}

Palabras Claves: Disposición técnica, fin de vida útil de los vehículos, uso racional, vehículos automotores.

\section{INTRODUCCIÓN}

En los últimos años el estudio de las cadenas productivas para el tratamiento de los vehículos al final de su vida útil toma cada vez más importancia, a juzgar por la presencia que tiene el tema en las publicaciones internacionales [1]. Los vehículos automotores al final de su vida útil (VFU) son una fuente de desechos reciclables y reutilizables. Entre los desechos reciclables 
se encuentran, en primer lugar, los desechos metálicos, tanto ferrosos como no ferrosos, que para Cuba significan la sustitución de importaciones y la posibilidad de exportación en el caso de los no ferrosos [2]. Las piezas y componentes en buen estado se reutilizan insertándose en el mercado como piezas de segunda mano.

Las cadenas productivas para el tratamiento de los VFU, en Cuba, recibieron un impulso como parte del programa de ahorro energético, principalmente la de tratamiento de vehículos de carga. Se aprobaron un grupo de instrumentos jurídicos regulando el proceso. Específicamente el Reglamento para la ejecución de las bajas de los vehículos automotores por carretera $\mathrm{y}$ sus medios traccionados, derivado de las Indicaciones Conjuntas SIME - MITRANS para la tramitación y concreción de las bajas de medios automotores, establece el procedimiento para materializar la baja de un vehículo de la flota. La decisión de dar baja de la flota a un vehículo determina el paso de este a la cadena de tratamiento de los VFU. Dicha resolución dispone que en la primera etapa del proceso de baja se realice la evaluación técnico - económica de la propuesta de vehículos para finalizar su vida útil. Para ello debe crearse una comisión integrada por representantes de las áreas técnica, operaciones y económica. Esta comisión deberá dictaminar acerca del estado técnico para la explotación segura y económica de los medios de transporte automotor y, con base a esta información, proponer al jefe de la entidad los vehículos a ser dados baja. Más recientemente se aprobó el procedimiento P-50507-01[3], que regula la aprobación de las bajas en el esquema empresarial subordinado al MITRANS. Este procedimiento, al igual que la resolución conjunta, parte de que las empresas deciden cuáles son los vehículos propuestos para finalizar su vida útil. Pero, ¿cuál es el momento oportuno para que esta comisión se cree y cuáles son los vehículos que deben ser valorados para finalizar su vida útil? no se aclara en regulación alguna.

El más reciente censo de vehículos de carga, realizado en el 2005, mostró que el $20 \%$ de los vehículos se encontraban paralizados por períodos superiores a seis meses. Esto significa la existencia en la flotas de transporte de carga que no están en condiciones de trabajar cuyo deterioro (dado por la oxidación fundamentalmente) se incrementa con el paso del tiempo, afectando las posibilidades de reutilización de piezas y la calidad de la chatarra y otros desechos reciclables de los vehículos. De ahí la importancia que tiene para la cadena productiva para el tratamiento de los VFU que la decisión de dar baja a un equipo de la flota se realice oportunamente.

\section{Considerando que:}

1. la racionalidad en el uso de los recursos es uno de los principios del desarrollo sostenible [4],

2. los vehículos de transporte son altos consumidores de recursos naturales en todo su ciclo de vida [5],

3. la política para el transporte aprobada por el VI Congreso del PCC establece el uso racional de los medios de transporte (L269) [6],

4. el Coeficiente de Disposición Técnica (CDT) caracteriza el estado técnico de un vehículo [7] y disminuye en la medida que se incrementa el deterioro del mismo.

Los autores del presente trabajo proponen la evaluación periódica de la racionalidad de la explotación de las flotas de transporte a partir del análisis del CDT y que el momento para valorar la posibilidad de dar baja a un vehículo de transporte de carga sea aquel en el que su uso sea irracional en flotas de transporte.

\section{EVALUACIÓN DE LA RACIONALIDAD DE LA EXPLOTACIÓN DE LAS FLOTAS}

\section{II.1 MODELO DE LA DISPOSICIÓN TÉCNICA MÍNIMA}

El uso de un vehículo se evalúa a partir del indicador Aprovechamiento del Parque $\left(\alpha_{a}\right)$. Este indicador depende a su vez del Coeficiente de Disposición Técnica $\left(\alpha_{t}\right)$, característico de la función Mantenimiento, y del Coeficiente de Salida $\left(\alpha_{\mathrm{s}}\right)$, propio de la función Operaciones. Los tres indicadores se relacionan, según Afanasiev [8], como se muestra a continuación:

$$
\alpha_{\alpha}=\alpha_{t} \cdot \alpha_{s}
$$

El CDT es un indicador que muestra la proporción del tiempo en que un vehículo se mantuvo en buen estado técnico para cumplir con su función [9]. Si se toma el estado técnico como factor para considerar el término de la vida útil de un vehículo en flotas de transporte de carga, el CDT será el indicador a analizar. Pero, para analizar un indicador es necesaria una referencia que posibilite la comparación y la evaluación.

El estado técnico de un vehículo depende de las características propias del vehículo y del régimen de trabajo al que es sometido. La referencia que se emplee para el análisis de la disposición técnica debe estar en función de los indicadores que caracterizan estos factores. La gran cantidad de combinaciones posibles entre los factores técnicos y económicos de influencia hace que la identificación de una referencia para la evaluación del estado del vehículo requiera de un modelo.

Para los efectos de esta investigación, se definió como condición límite de operación racional a la resultante de la relación matemática que se establece entre el estado técnico y el resultado económico de la operación de los vehículos (Erro! Fonte de referência não encontrada.).

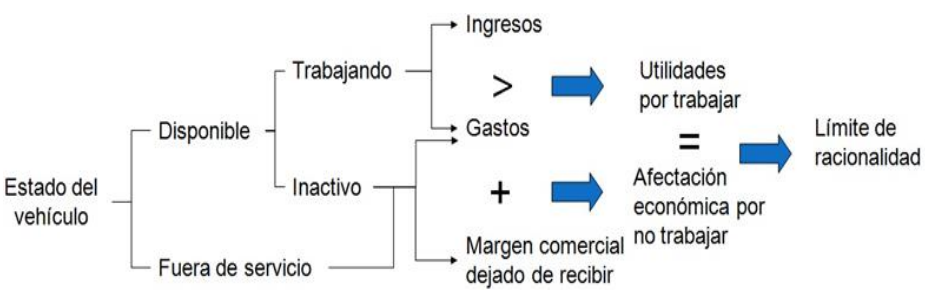

Figura 1: Relación entre el estado de un vehículo y el resultado económico.

Fuente: Los autores, (2018).

Esta relación se obtuvo modificando la Condición Económica de Mínimo utilizada por Kochnov y Basté [10] para la determinación del recurso medio entre reparaciones generales de los vehículos.

La combinación de esta condición límite con la forma de cálculo del CDT permite obtener la función matemática siguiente [11]:

$$
\alpha_{t_{M I N}}=\frac{b}{\alpha_{s}(b+a)}
$$

Donde:

$\alpha_{t M I N}$ : Coeficiente de Disposición Técnica Mínimo. 
$\alpha_{\mathrm{s}}$ : Coeficiente de salida.

$a$ : Utilidad diaria por la operación de un vehículo [\$/d].

$b$ : Afectación económica diaria que genera un vehículo por no trabajar $[\$ / d]$.

La identificación de los parámetros de la transportación de cargas asociados a los términos $a$ y $b$ permite obtener la relación siguiente [11]:

$$
\alpha_{t_{M I N}}=\frac{V_{d}-v \cdot l_{t_{d}}-F_{d}}{2 \alpha_{s}\left(V_{d}-v \cdot l_{t_{d}}\right)}
$$

Donde:

$V_{d}$ : Ingreso medio diario $[\$ / \mathrm{d}]$.

$v$ : Costo variable unitario $[\$ / \mathrm{km}]$.

$l_{t d}$ : Distancia promedio recorrida al día $[\mathrm{km} / \mathrm{d}]$.

$F_{d}$ : Costo fijo diario $[\$ / \mathrm{d}]$.

Como se puede observar, la expresión (3) relaciona los indicadores que determinan el aprovechamiento de los vehículos según la expresión (1).

\section{II.1.1 PROCEDIMIENTO DE EVALUACIÓN}

Para evaluar la racionalidad de la operación de los vehículos de carga se debe:

1. Agrupar la flota atendiendo a las condiciones de explotación. La evaluación de la racionalidad de la operación de una flota se sustenta en el análisis individual de los vehículos. No obstante, se presupone que vehículos iguales operando bajo las mismas condiciones de explotación tienen igual $\alpha_{t M I N}$. Por tanto, es necesario identificar aquellos factores del entorno en los que operan los vehículos de carga que resultan relevantes para la evaluación de la racionalidad de la flota. O sea, aquellos factores que determinan los grupos de vehículos en los cuales se puede considerar que los parámetros de la disposición técnica mínima se comportan de manera similar. Entre estos factores se encuentran: la edad de los vehículos, el régimen de

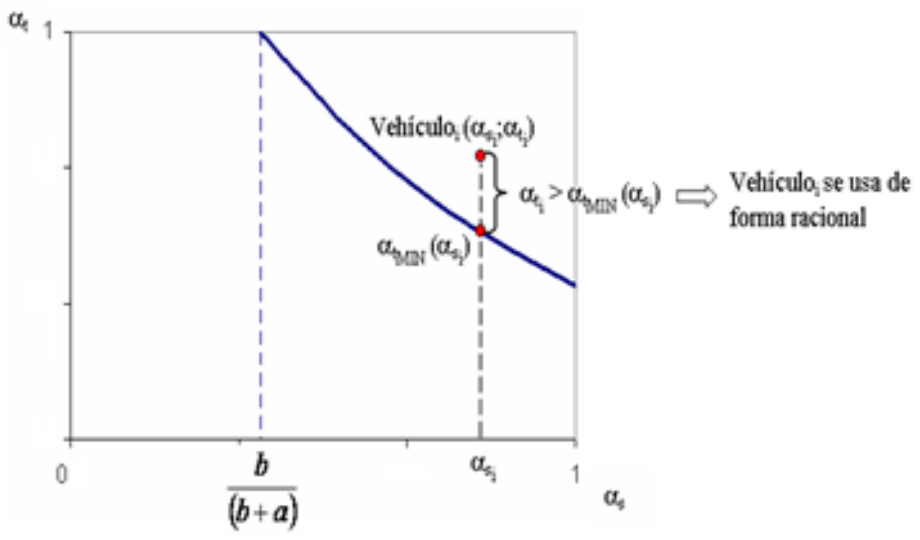

a. Vehículo operado de forma racional

Figura 3. Condiciones para la identificación de los vehículos operados de forma racional e irracional.

Fuente: Los autores, (2018). trabajo, tipo de carga que transportan, características de las vías por las que circulan, entre otros [12].

2. Identificar los parámetros del modelo de la disposición técnica mínima. Monitorear un indicador significa disponer de la referencia con anterioridad. El momento de la planificación económica, que se realiza en todas las empresas cubanas previo al inicio de un período económico, es el oportuno para obtener los valores de los parámetros del modelo.

3. Determinar la constante de la función. Al sustituir los parámetros $\left(V_{d}, v, l_{t d}\right.$ y $\left.F_{d}\right)$ en la función se obtiene una hipérbole. En la representación gráfica de la función (Figura 2) se puede distinguir la zona de operación racional (área por encima de la curva) y la zona de operación irracional (área por debajo de la curva).

4. Identificar los vehículos operados de forma racional e irracional. La determinación de la racionalidad de la operación de los vehículos se realiza a partir de la evaluación de la condición de uso racional mostrada en la $\quad$ Figura 3 para cada vehículo en el grupo. Esto permite identificar aquellos que son operados de forma racional y aquellos que no lo son.

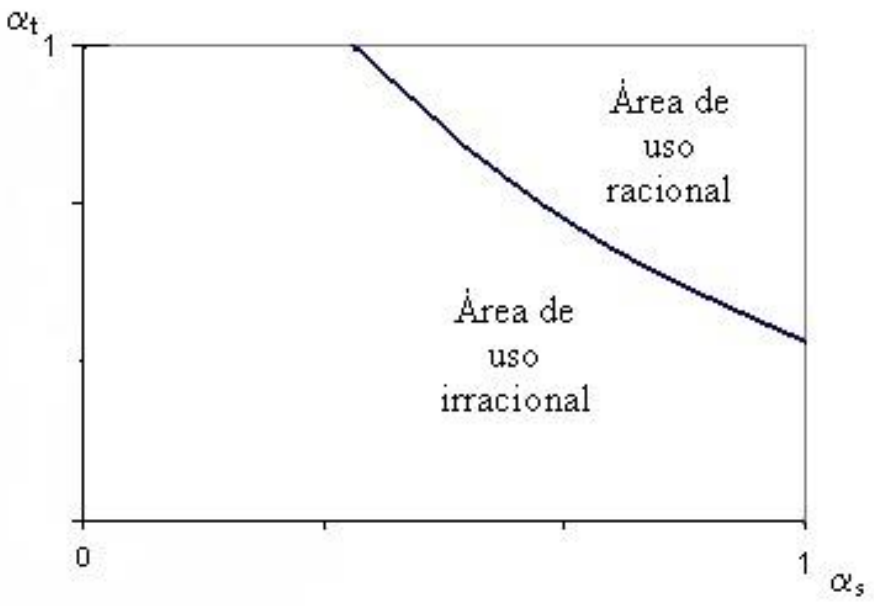

Figura 2: Comportamiento del CDT Mínimo en función del Coeficiente de Salida.

Fuente: Los autores, (2018).

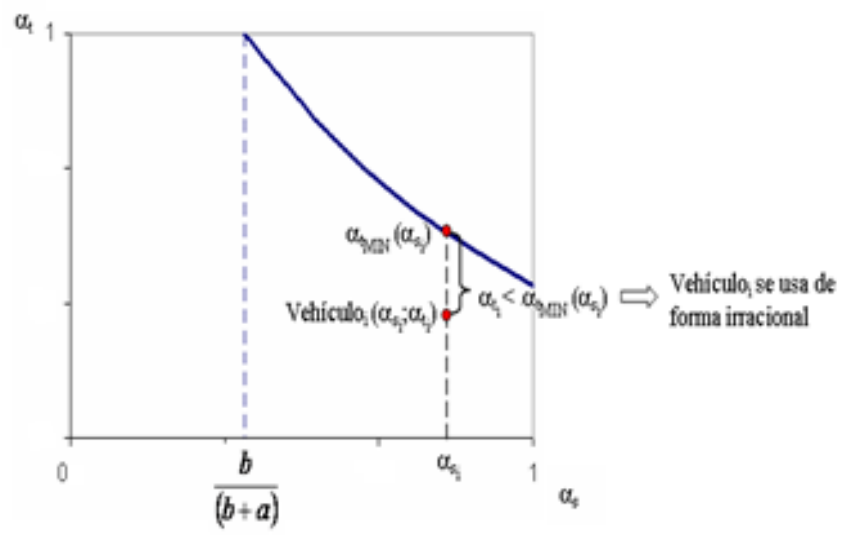

b. Vehículo operado de forma

imacional 


\section{RESULTADOS Y DISCUSIÓN}

Para la aplicación del modelo se escogió una flota de 40 cuñas tractivas pertenecientes a una flota de transportación de cereales. El análisis del contexto operacional arrojó que el criterio relevante para la agrupación de la flota es la tecnología. De esta manera se obtuvo dos grupos homogéneos (GH) con la composición que muestra la Tabla 1.

Se calcularon los valores de $V_{d}, v, l_{t d}$ y $F_{d}$ para cada $\mathrm{GH}$ a partir del plan económico aprobado. Los resultados se muestran en la Tabla 2. Esto permitió obtener las curvas de la disposición técnica mínima (Figura 4).

$\mathrm{GH} 1$

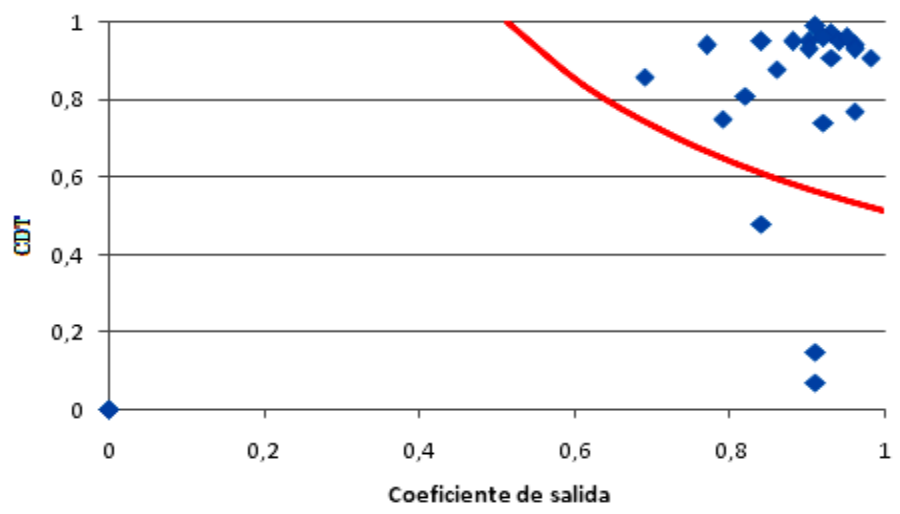

Figura 4. Comportamiento de los vehículos por GH.

Fuente: Los Autores, (2018).

La ubicación de los pares coordenados $\left(\alpha_{\mathrm{s}} ; \alpha_{\mathrm{t}}\right)$ correspondiente a cada equipo en los gráficos arrojó que el $75 \%$ de los vehículos se están explotando de forma racional, mientras que el $25 \%$ se explota de forma irracional. Estos resultados permiten a la empresa operadora de la flota valorar la posibilidad de decidir el fin de la vida útil de los vehículos cuya operación es irracional y con ello dar paso a la etapa de fin de vida del vehículo.

\section{CONCLUSIONES}

La decisión de finalizar la vida útil de un vehículo de carga en una flota debe tomarse de manera oportuna, de forma tal que permita durante el tratamiento de fin de vida un mejor aprovechamiento de sus desechos reutilizables y reciclables.

El Coeficiente de Disposición Técnica Mínimo se obtiene de la combinación del Coeficiente de Disposición Técnica y la condición límite de uso racional y relaciona los indicadores que determinan el aprovechamiento del vehículo.

El uso del Coeficiente de Disposición Técnica Mínima como referencia durante el análisis de la disposición técnica permite identificar los vehículos que son operados de forma racional y aquellos que no lo son. Los vehículos cuya operación es irracional deberán valorarse para su posible baja de la flota y con ello su tránsito a la cadena productiva de tratamiento de VFU.

\section{REFERENCIAS}

[1] JUNWEI GAN, Q. C. y ZHENGGANG HE, W. C. "Literature Review and Prospect on the End-of-Life Vehicles
Tabla 1: Composición del los GH.

\begin{tabular}{|c|c|c|c|c|}
\hline Grupo & $\begin{array}{c}\text { Capacidad } \\
\text { de carga (t) }\end{array}$ & $\begin{array}{c}\text { Peso } \\
\text { bruto(t) }\end{array}$ & $\begin{array}{c}\text { Tiempo en } \\
\text { operación }\end{array}$ & Cantidad \\
\hline GH1 & 30 & 16 & 7 & 27 \\
\hline GH2 & 30 & 25 & 4 & 13 \\
\hline \multicolumn{4}{|c}{ Total } \\
\hline
\end{tabular}

Fuente: Los Autores, (2018).

Tabla 2: Valores de los parámetros del modelo por grupo homogéneo.

\begin{tabular}{|l|l|c|c|c|l|}
\hline Grupo & $V_{d}$ & $l_{t d}$ & $V$ & $F_{d}$ & Constante \\
\hline $\mathrm{GH} 1$ & 1043,45 & 407,34 & 0,3170 & 25,43 & 0,5139 \\
\hline $\mathrm{GH} 2$ & 989,30 & 391,97 & 0,3511 & 52,46 & 0,5307 \\
\hline
\end{tabular}

Fuente: Los Autores, (2018).

\section{$\mathrm{GH} 2$}

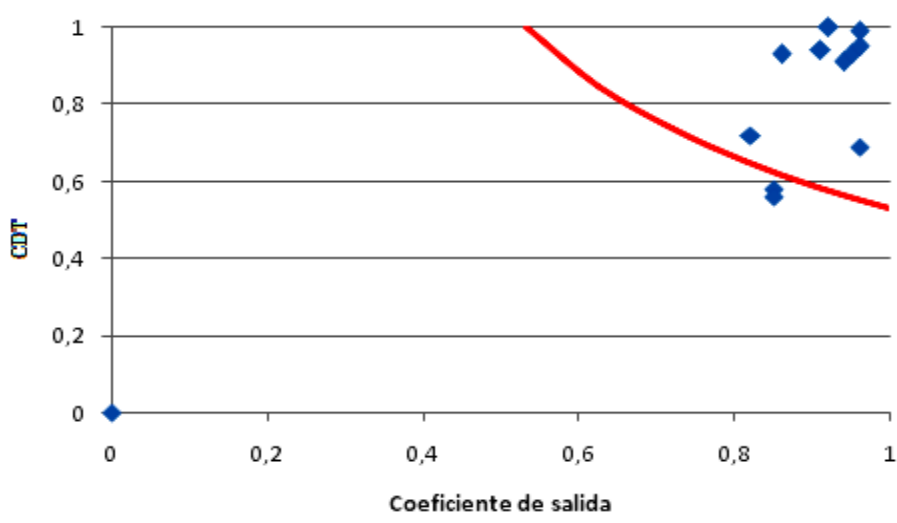

Reverse Logistics". Advanced Materials Research. 2014, nº 878, p. 66-74. Disponible en: http://search.ebscohost.com/login.aspx?direct=true\&db=aci\&AN =94759714\&lang=es\&site=ehost-live. ISSN 16628985.

[2] Cuba apuesta al Reciclaje. Resumen de la intervención de los directivos principales de la Unión de Empresas de Recuperación de Materias Primas en la Mesa Redonda del 26 de junio de 2013. La Habana: publicado el: 27-6-2013 de 2013, última actualización: 27-6-2013. vol. 2013, Disponible en: http://www.cubadebate.cu/categoria/temas/economia-temas/.

[3] MINISTERIO DEL TRANSPORTE. Procedimiento para la tramitación, ejecución y aprobación de las bajas técnicas de vehículos automotores. La Habana: 2013, vol. P-50507-01,

[4] AYES AMETller, G. N. Desarrollo Sostenible y sus retos. La Habana: Editorial Ciencia y Técnica, 2004. 191 p. p ISBN 959-05-0411-6.

[5] OBSERVATORIO INDUSTRIAL DEL SECTOR FABRICANTES DE AUTOMÓVILES Y CAMIONES. Evaluación básica de análisis de ciclo de vida de la fabricación, uso y fin de vida de los automóviles y camiones en España. Fedit. 24 de enero de 2012 de 2012.

[6] VI CONGReso PCC. Lineamientos de la Política Económica y Social del Partido y la Revolución. La Habana: 2011, 38 p. 
[7] MITRANS. Índices técnico económicos del transporte terrestre. Análisis de métodos y cronometraje. La Habana: Imprenta revolucionaria, 1966. $\mathrm{p}$

[8] AFANASIEV, L. L., et al. Sistema único de transportación y transportaciones por vehículos automotores. Editado por: Transport. 2da ed. Moscú: 1984. 333 p. p

[9] CAMARGO, J. Indicadores del Transporte. La Habana: Editorial Ciencias Sociales, 1989. p Mitrans

[10] KOCHNOV, N. y BASTÉ, J. Reparación de los automóviles. vols. 2. La Habana, Cuba: EMPSES, 1986. vol. 1, p

[11] PENABAD SANZ, L. Modelo para la determinación de la disposición técnica mínima de flotas de transporte de carga. Tutor: Rodríguez Ramos, P. A. Tesis en opción al título de Especialista de Postgrado en Gestión Integral del Transporte, Mención Transporte Automotor, Ingeniería del Transporte. cujae, La Habana. 2011.

[12] CARRILlo GAlVEZ, A y PENABAD SANZ, L. Contexto operacional en flotas de transporte: Características e importancia. En 18 Convención Científica de Ingeniería y Arquitectura. La Habana. 21-25 de noviembre. 2016. 